\title{
Prostatectomy Provides Better Symptom-Free Survival Than Radiotherapy Among Patients With High-Risk or Locally Advanced Prostate Cancer After Neoadjuvant Hormonal Therapy
}

\author{
Sung Han Kim ${ }^{1}$, Mi Kyung Song ${ }^{2,3}$, Weon Seo Park ${ }^{4}$, Jae Young Joung ${ }^{1}$, \\ Ho Kyung Seo ${ }^{1}$, Jinsoo Chung ${ }^{1}$, Kang Hyun Lee ${ }^{1}$
}

\author{
${ }^{1}$ Department of Urology, Center for Prostate Cancer, National Cancer Center Hospital, Goyang, Korea \\ ${ }^{2}$ Biometrics Research Branch and Biostatistics Collaboration Unit, National Cancer Center Hospital, Goyang, Korea \\ ${ }^{3}$ Health Insurance Policy Research Institute, National Health Insurance Service, Wonju, Korea \\ ${ }^{4}$ Department of Pathology, Center for Prostate Cancer, National Cancer Center Hospital, Goyang, Korea
}

Purpose: The purpose of this study is to compare the radiation therapy (RT) and radical prostatectomy (RP) of high-risk or locally advanced prostate cancer (PC) patients after neoadjuvant hormonal therapy (NHT). Materials and Methods: This retrospective study evaluated patients underwent RT (42 patients) or RP (152 patients) after NHT at a single center during 2003-2014. Times to biochemical recurrence (BCR), pelvic local recurrence (PLR), metastasis, clinical painful symptom progression (CPSP), castration-resistant PC (CRPC), and overall survival were compared between the RT and RP groups, after adjustment for TN stage, using the Kaplan-Meier method and log-rank test.

Results: Significant inter-group differences were observed for age, Gleason score, initial PSA, and clinical and pathological T stages (all $p<0.05$ ). During a median follow-up of 71.7 months, the overall incidences of BCR, PLR, metastasis, CPSP, CRPC, and death were $49.5 \%, 16.5 \%, 8.3 \%, 7.7 \%, 7.7 \%$, and $17.5 \%$, respectively. The median times to BCR were 100 months for RT and 36.2 months for RP $(p=0.004)$, although the median times were not reached for the other outcomes (all $p>0.05$ ). The independent predictor of CPSP was RP (hazard ratio, $0.291 ; \mathrm{p}=0.013$ ).

Conclusions: Despite significantly different baseline parameters, RP provided better CPSP-free survival than RT among patients with localized high-risk or locally advanced PC. (Korean J Urol Oncol 2018;16:126-134)

Key Words: Neoadjuvant $\cdot$ Hormone $\cdot$ Prostate cancer $\cdot$ Recurrence $\cdot$ Risk factor

Received November 1, 2018, Revised November 28, 2018,

Accepted December 7, 2018

Corresponding Author: Kang Hyun Lee

Department of Urology, Center for Prostate Cancer, Research Institute and Hospital of National Cancer Center, 323 Ilsan-ro, Ilsandong-gu, Goyang 10408, Korea

E-mail: uroonco@ncc.re.kr

Tel: +82-31-920-2799, Fax: +82-31-920-1676

ORCID code: https://orcid.org/0000-0003-0041-2243

- This study was supported by the Korean National Cancer Center Grants (1810021-1).

\section{INTRODUCTION}

Prostate cancer (PC) is the most prevalent cancer and the second leading cause of cancer-related death among men. ${ }^{1}$ Thus, PC is associated with a major health burden and its heterogeneity produces diverse clinical outcomes, which makes it difficult to identify patients with high risks of recurrence and progression. Thus, stratifying patients into low-, intermediate-, and high-risk (or very high risk) groups based on different risk factors have been suggested (e.g., based on their initial pros-

(i) (3) This is an Open Access article distributed under the terms of the Creative Commons Attribution Non-Commercial License (http://creativecommons.org/licenses/by-nc/4.0/) which permits unrestricted non-commercial use, distribution, and reproduction in any medium, provided the original work is properly cited. 2018 (C) Copyright The Korean Urological Oncology Society and The Korean Prostate Society. All Rights Reserved. 
tate-specific antigen [PSA] level, Gleason score, or disease stage). ${ }^{2,3}$

According to version 3.2016 of the National Comprehensive Cancer Network guidelines for PC, a high risk of PC is considered present for patients with PSA levels of $\geq 20 \mathrm{ng} / \mathrm{mL}$, Gleason scores of $\geq 8$, or clinical T3 tumors. ${ }^{3}$ The treatment options for high-risk PC include external beam radiation therapy (RT) plus androgen-deprivation therapy (ADT) with/without brachytherapy or radical prostatectomy plus pelvic lymph nodal dissection (RP). Both RT and RP provide similar local control rates and improve survival and quality of life.,

Neoadjuvant hormonal therapy (NHT) can help improve recurrence-free survival among men with advanced PC who receive ADT and RP. ${ }^{6}$ This is because NHT reduces the tumor's size, improves surgical curability by inhibiting the growth of PC cells, and promotes down-staging, lower regional metastasis rates, better organ confinement, and decreased lymph node involvement. ${ }^{7}$ However, these effects do not translate into better overall survival (OS) or disease-free survival. ${ }^{6}$ Nevertheless, among localized high-risk or locally advanced PC cases, the time to biochemical recurrence (BCR) after NHT (i.e., the time from the PSA nadir after treatment) can influence the long-term BCR-free outcomes after RT among patients with PC. ${ }^{8}$

No large-scale prospective randomized clinical trials have compared the existing treatment options with NHT, and there are no optimal management strategies for patients with high-risk PC. Moreover, previous studies comparing RP and RT are difficult to interpret, because of the presence of comorbidities, biased treatment selection criteria, incomplete follow-up data, varied treatment protocols, and reliance on surrogate endpoints. ${ }^{9}$ Therefore, we aimed to compare RT and RP for patients with high-risk PC after NHT using various outcomes: BCR-free survival (BCRFS), pelvic local recurrence-free survival (PLRFS), metastasis-free survival (MFS), clinical pain-free survival (CPFS), castration-resistant PC (CRPC), and OS.

\section{MATERIALS AND METHODS}

This study was performed in accordance with the World Medical Association's Declaration of Helsinki-Ethical Principles for Medical Research Involving Human Subjects. The study's retrospective protocol was approved by the Institutional Review Board of the Research Institute and Hospital National
Cancer Center (NCCNCS 05-049). All enrolled patients provided written informed consent.

During 2000-2015, 762 consecutive patients with high-risk PC were treated at the Center for Prostate Cancer (National Cancer Center, Korea). High-risk PC was defined as cases with PSA levels of $\geq 20 \mathrm{ng} / \mathrm{mL}$, Gleason scores of $\geq 8$, or clinical T3 tumors. ${ }^{2,3}$ The exclusion criteria were missing information (e.g., loss to follow-up or a follow-up duration of $<1$ year), and a history of only NHT or RP. A total of 613 cases were reviewed, and 194 cases with complete information were included (152 patients who received NHT followed by RP and 42 patients who received NHT followed by external beam radiotherapy). All RP specimens were independently reviewed by a uropathologist with 15 years of experience (WSP), according to the guidelines of the 2005 International Society of Urological Pathology consensus conference. ${ }^{10}$

The RP group received leuprolide $(11.25 \mathrm{mg}$ ) or $10.8 \mathrm{mg}$ of goserelin acetate every 3 months for 3-6 months before the RP. All patients in the RP group underwent the same standard pelvic lymphadenectomy procedure, which included removal of the bilateral obturator lymph nodes with or without the iliac lymph nodes. The RT group received ADT (a luteinizing hormone-releasing hormone agonist and an antiandrogen) for at least 9-12 months (median, 12 months) before undergoing EBRT (using a 3-dimensional conformal RT [3D-CRT] technique) and subsequent adjuvant hormonal therapy (HT) for 1-2 years. The clinical target volume included the entire prostate, the bases of the seminal vesicles, and the pelvic lymph nodes. The planning target volume was defined using a safety margin of $10 \mathrm{~mm}$ in most directions, with a posterior margin of $6 \mathrm{~mm}$. The total radiation dose was 70-76 Gy delivered in 2-Gy fractions five times per week. ${ }^{11}$

All patients underwent serum PSA and testosterone monitoring every 3 months for 5 years and then every 6 months thereafter. Pretreatment serum PSA levels were measured within 1 month before the RP or EBRT. In the RP group, disease recurrence or PSA failure (BCR) was defined as a consecutive increase of serum PSA level of $>0.2 \mathrm{ng} / \mathrm{mL}$. If the PSA level does not drop $<0.2$, then the consecutive increase of PSA level after the lowest PSA level was the date of BCR. In the RT group, BCR was defined according to the 2006 consensus statement by the American Society of Therapeutic Radiation and Oncology, which defined biochemical failure after EBRT as PSA levels rising by $\geq 2.0 \mathrm{ng} / \mathrm{mL}$ above the nadir PSA level. ${ }^{12}$ 
A

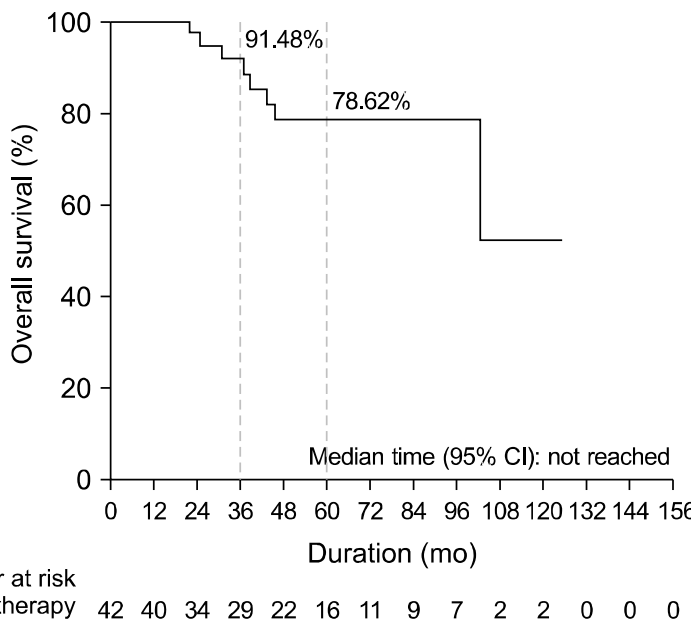

B

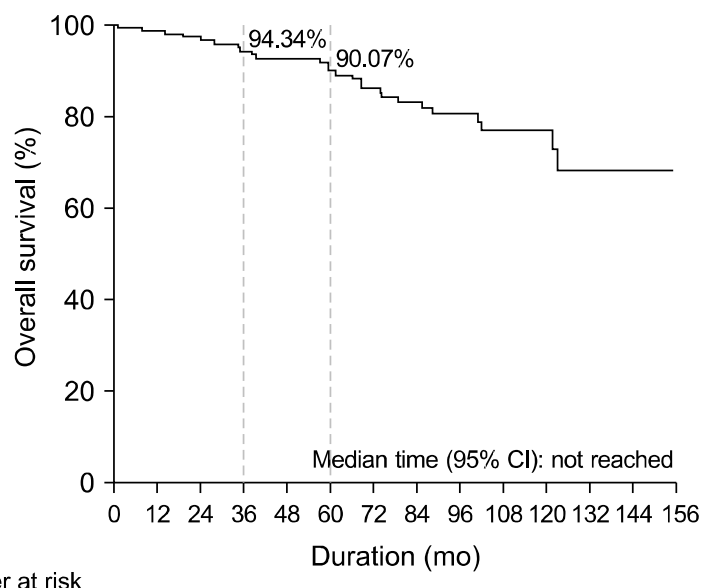

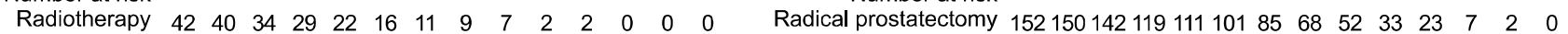

Fig. 1. Overall 3-year and 5-year survival curves for the radiotherapy (A) and radical prostatectomy (B) groups. CI: confidence interval.

Table 1. The patients' baseline clinicopathological characteristics

\begin{tabular}{|c|c|c|c|c|}
\hline Characteristic & Total $(n=194)$ & RT $(n=42)$ & $\mathrm{RP}(\mathrm{n}=152)$ & p-value \\
\hline Age $(\mathrm{yr})$ & $68.0(47.0-81.0)$ & $70.5(54.0-81.0)$ & $67.0(47.0-78.0)$ & 0.001 \\
\hline Gleason score sum & & & & 0.243 \\
\hline $\mathrm{GS} \leq 6$ & $35(18.0)$ & $5(11.9)$ & $30(19.7)$ & \\
\hline $\mathrm{GS}>6$ & $159(82.0)$ & $37(88.1)$ & $122(80.3)$ & \\
\hline Initial PSA & $30.6(2.7-261.8)$ & $21.3(2.7-147.3)$ & $34.0(3.2-261.8)$ & 0.028 \\
\hline cT stage & & & & $<0.001$ \\
\hline $\mathrm{T} 2(2 \mathrm{a}, 2 \mathrm{~b}, 2 \mathrm{c})$ & $90(46.4)$ & $9(21.4)$ & $81(53.3)$ & \\
\hline $\mathrm{T} 3(3 \mathrm{a}, 3 \mathrm{~b}, 3 \mathrm{c}, \mathrm{p} 3 \mathrm{~b})$ & $95(49.0)$ & $29(69.1)$ & $66(43.4)$ & \\
\hline $\mathrm{T} 4(4, \mathrm{p} 4)$ & $9(4.6)$ & $4(9.5)$ & $5(3.3)$ & \\
\hline $\mathrm{cN}$ stage & & & & 0.259 \\
\hline N0 & $173(89.2)$ & $40(95.2)$ & $133(87.5)$ & \\
\hline N1 & $21(10.8)$ & $2(4.8)$ & $19(12.5)$ & \\
\hline cTN stage & & & & $<0.001$ \\
\hline $\mathrm{cT} 1 \mathrm{~N} 0+\mathrm{cT} 2 \mathrm{~N} 0$ & $87(44.9)$ & $9(21.4)$ & $78(51.3)$ & \\
\hline cT3N0+cT4N0 & $86(44.3)$ & $31(73.8)$ & $55(36.2)$ & \\
\hline $\mathrm{LN}^{+}$ & $21(10.8)$ & $2(4.8)$ & $19(12.5)$ & \\
\hline pTN stage & & & & NA \\
\hline урт0 & $4(2.6)$ & - & $4(2.6)$ & \\
\hline ypT1N0+ypT2N0 & $94(61.8)$ & - & $145(98.0)$ & \\
\hline ypT3N0+ypT4N0 & $52(34.2)$ & - & $3(2.0)$ & \\
\hline Positive surgical margins & $7(4.6)$ & - & $7(4.6)$ & NA \\
\hline Follow-up duration (mo) & $71.7(1.4-155.0)$ & $49.8(9.4-124.9)$ & $79.2(1.4-155.0)$ & $<0.001$ \\
\hline Biochemical recurrence & $96(49.5)$ & $11(26.2)$ & $85(55.9)$ & $<0.001$ \\
\hline Local pelvic recurrence & $32(16.5)$ & $5(11.9)$ & $27(17.8)$ & 0.365 \\
\hline Metastasis & $16(8.3)$ & $3(7.1)$ & $13(8.6)$ & 1.000 \\
\hline Clinically painful symptoms & $15(7.7)$ & $6(14.3)$ & $9(5.9)$ & 0.098 \\
\hline Castration resistant & $15(7.7)$ & $1(2.4)$ & $14(9.2)$ & 0.199 \\
\hline Death & $34(17.5)$ & $8(19.1)$ & $26(17.1)$ & 0.770 \\
\hline
\end{tabular}

Values are presented as median (range) or number (\%).

RT: radiotherapy, RP: radical prostatectomy, GS: Gleason score, PSA: prostate-specific antigen, NA: not available. 
Data regarding baseline characteristics were presented as median and range (continuous variables) or frequency and percentage (categorical variables). Continuous and categorical variables were compared using the Mann-Whitney U-test and the chi-square test or Fisher exact test, respectively. Survival analyses were performed for the 2 groups' BCRFS, PLRFS, CPFS, MFS, CRPC, and OS. The outcome of clinical pain included flank and lower perineal pain, dysuria, hematuria, urinary retension, urgency related lower abdominal pain, scrotal pain including testicular and epididymal pain, and so on which affected patients to visit either the Emergency Department or outpatient clinics for the pain relief and therapeutic intervention. The pain scale and the pain description including narcotic analgesics were reviewed retrospectively from the medical charts to evaluate the grade of pain scale from pain-specialized anesthesiologists and clinicians.

These outcomes were generally calculated from the surgery for the RP group and from the end of RT for the RT group. However, if the period between NHT and adjuvant HT was $<1$ month in the RT group, the time to BCR was calculated from the start of RT, rather than from the end of RT. The last follow-up was used to calculate the duration for censored events. Survival curves were plotted using the Kaplan-Meier method and compared using the log-rank test. Multivariable Cox proportional hazard models were used to identify associations with the survival outcomes. Due to the presence of zero cells, Firth correction and profile-likelihood confidence limits were used to properly estimate the effects. Two-tailed p-values of $<0.05$ were considered statistically significant. Statistical analyses were performed using SAS ver. 9.4 (SAS Institute Inc., Cary, NC, USA) and R ver. 3.1.2 (R Foundation for Statistical Computing, Vienna, Austria; http://www.R-project.org).

\section{RESULTS}

During a median follow-up of 71.7 months (range, 1.4-155.0 months), the 3- and 5-year OS rates were $91.5 \%$ and $78.6 \%$ for RT, compared to $94.3 \%$ and $90.1 \%$ for RP without reaching the median OS (Fig. 1). The median patient age was 66 years (range, 44-89 years). The overall incidences of BCR, PLR, metastasis, CPSP, CRPC, and death were $49.48 \%, 16.49 \%$, $8.25 \%, 7.73 \%, 7.73 \%$, and $17.53 \%$, respectively (Table 1). All patients in the RP group were evaluated for pathological response, which revealed ypT0 tumors $(2.6 \%, \mathrm{n}=4)$, ypT1-2 tu- mors $(97.97 \%, \mathrm{n}=145)$, and $\geq \mathrm{ypT3}$ tumors $(2.03 \%, \mathrm{n}=3)$. Seven patients $(4.6 \%)$ had positive surgical margins, and 96 patients $(49.48 \%)$ received adjuvant ADT or RT. The clinicopathological characteristics of the RT and RP groups are shown in Table 1, with significant differences detected for age, initial PSA level, cT stage, cTN stage, follow-up duration, and BCR (all p<0.05).

At the time of the analysis, 8 patients in the RT group (19.1\%) and 26 patients in the RP group (17.1\%) had died (Table 1). The causes of death were PC, colorectal cancer, hepatocellular carcinoma, cerebral hemorrhage, and chronic heart failure. One patient from the RP group committed suicide. Multivariate analysis of CPFS showed that only RP was a significant predictor (hazard ratio, 0.291; $\mathrm{p}=0.045$ ) (Table 2).

At the time of the analysis, BCR had occurred in 11 patients in the RT group (26.2\%) and 85 patients in the RP group (55.9\%), with 3-year BCRFS rates of $86.5 \%$ in the RP group and $89.4 \%$ in the RT group ( $\mathrm{p}=0.004$ ) (Fig. 2). Comparisons of the BCRFS, PLRFS, MFS, CPFS, CRPC, and OS outcomes only revealed significant differences in BCRFS (36.2 months for RP vs. 100.0 months for RT, p=0.004) and CPFS (median not reached for both groups, $\mathrm{p}=0.013$ ) (Fig. 2).

Table 2. Multivariable Cox regression proportional analysis for clinical pain-free survival

\begin{tabular}{lcc}
\hline \multicolumn{1}{c}{ Variable } & HR $(95 \%$ CI $)$ & p-value \\
\hline Group & 1 (Reference) & \\
RT & 0.291 (0.092-0.959) & 0.045 \\
RP & $0.959(0.894-1.034)$ & 0.266 \\
Age & 1 (Reference) & \\
Gleason score & 6.376 (0.802-824.451) & 0.227 \\
GS $\leq 6$ & 1.006 (0.992-1.016) & 0.300 \\
GS $>7$ & 1 (Reference) & \\
Initial PSA & 1.864 (0.613-6.099) & 0.308 \\
cT stage & 3.330 (0.302-21.348) & 0.272 \\
T2 (2a, 2b, 2c) & 1 (Reference) \\
T3 (3a, 3b, 3c, p3b) & \\
T4 & 0.878 (0.079-4.638) & 0.896 \\
cN stage & 0.985 (0.861-1.065) & 0.749 \\
N0 & & \\
N1 & & \\
Neoadjuvant treatment & \\
duration &
\end{tabular}

HR: hazard ratio, RT: radiotherapy, RP: radical prostatectomy, PSA: prostate-specific antigen, GS: Gleason score. 
A

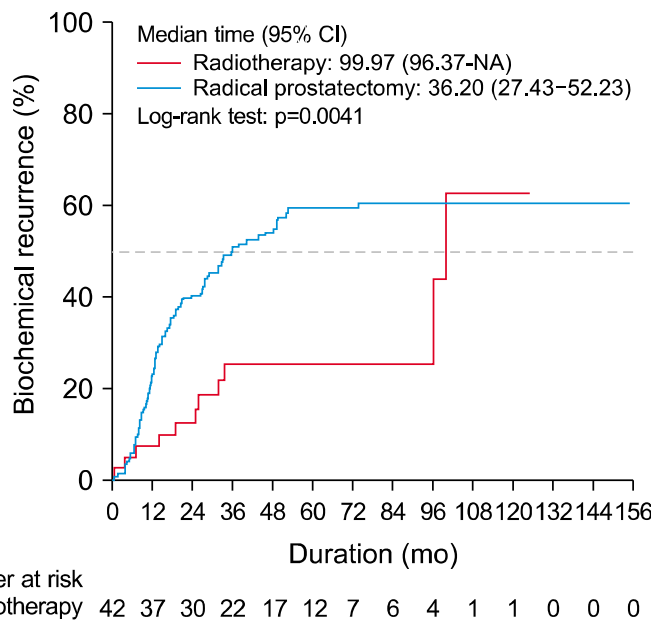

$\begin{array}{lllllllllllllll}\text { Radiotherapy } & 42 & 37 & 30 & 22 & 17 & 12 & 7 & 6 & 4 & 1 & 1 & 0 & 0 & 0\end{array}$

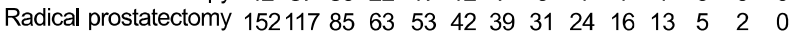

\section{C}

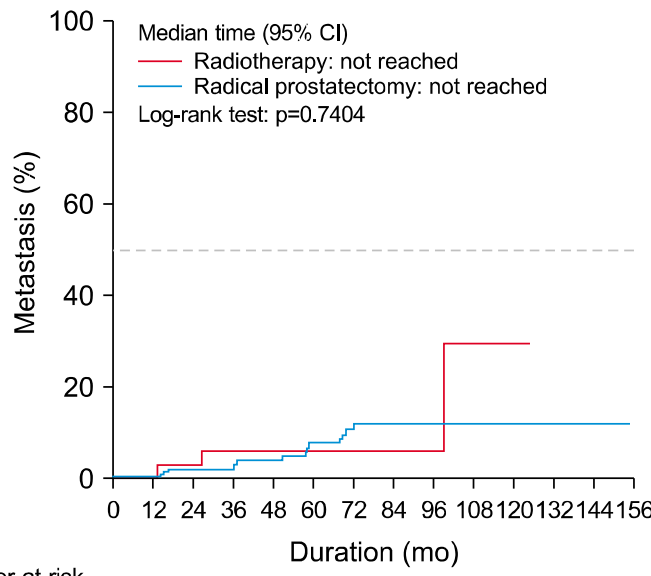

Number at risk

$\begin{array}{lllllllllllllll}\text { Radiotherapy } & 42 & 40 & 33 & 27 & 21 & 16 & 11 & 9 & 7 & 1 & 1 & 0 & 0 & 0\end{array}$

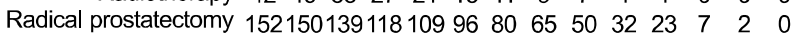

\section{E}

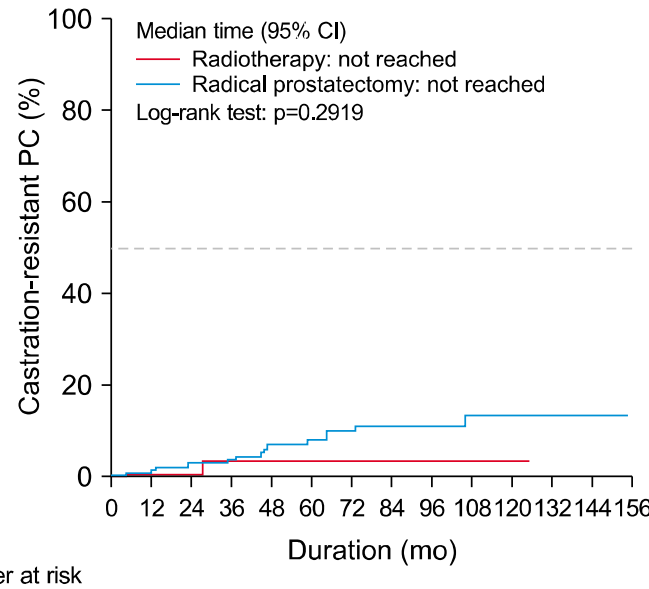

Number at risk

Radiotherap
B

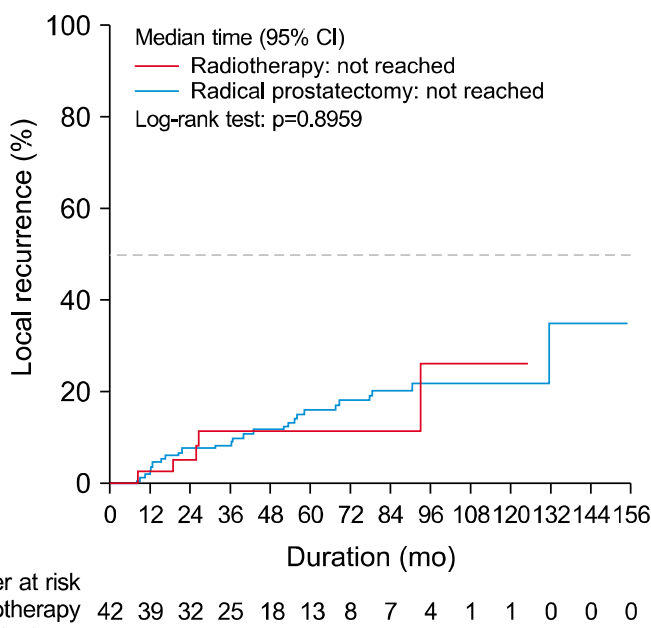

$\begin{array}{lllllllllllllll}\text { Radiotherapy } & 42 & 39 & 32 & 25 & 18 & 13 & 8 & 7 & 4 & 1 & 1 & 0 & 0 & 0\end{array}$

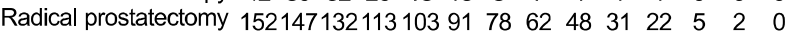

D

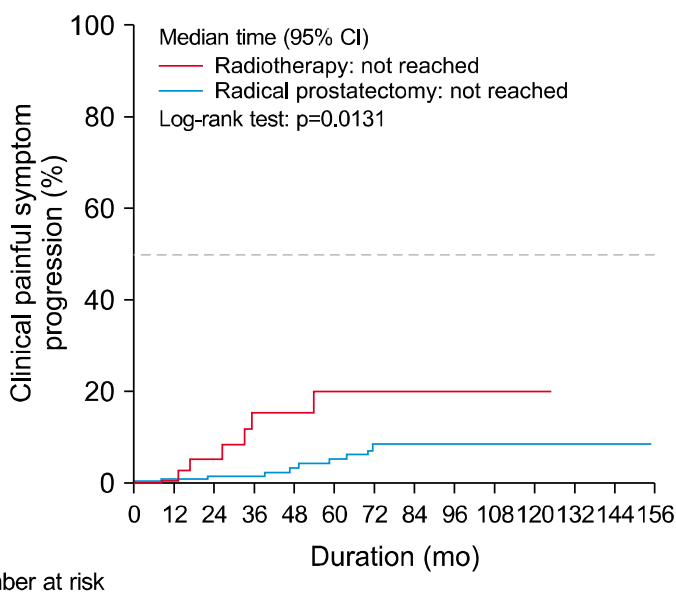

$\begin{array}{lllllllllllllll}\text { Radiotherapy } & 42 & 40 & 32 & 25 & 21 & 14 & 10 & 8 & 6 & 1 & 1 & 0 & 0 & 0\end{array}$

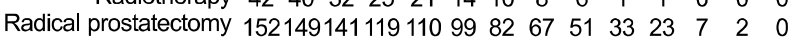

$\mathbf{F}$

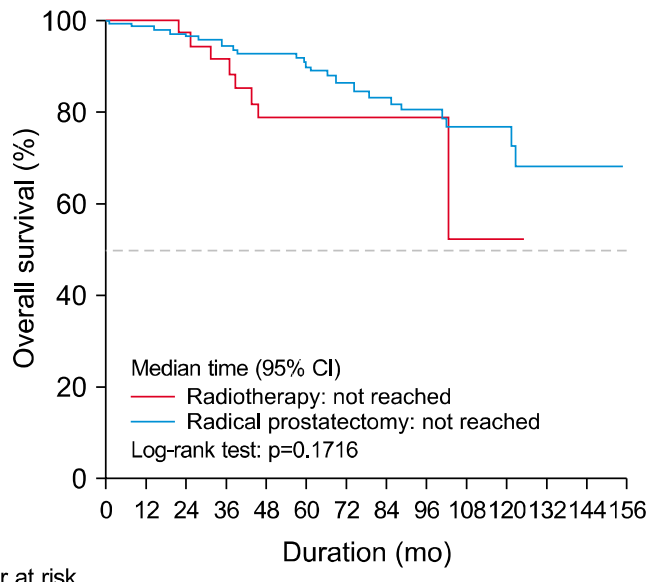

Number at risk

Radiotherapy $42 \quad 40 \begin{array}{llllllllllll}34 & 29 & 22 & 16 & 11 & 9 & 7 & 2 & 2 & 0 & 0 & 0\end{array}$

Fig. 2. Kaplan-Meier curves for biochemical recurrence (A), local recurrence (B), metastasis (C), clinically painful symptoms (D), castration-resistant prostate cancer $(\mathrm{E})$, and overall survival $(\mathrm{F})$ in the prostatectomy and radiotherapy groups. CI: confidence interval, NA: not assessed. 


\section{DISCUSSION}

Global reports have indicated that $<20 \%$ of patients with newly diagnosed PC have locally advanced or localized highrisk PC (i.e., T3 stage), ${ }^{13,14}$ in which the tumor has spread beyond the prostatic capsule or grown into the seminal vesicles. However, preoperative clinical staging is unreliable and inaccurate for detecting local tumor extension (50\% tumors have spread outside the prostate and $18 \%$ of tumors are confined to the prostate), which results in high rates of recurrence and disease progression. ${ }^{15}$ For high-risk or locally advanced PC, the 2016 NCCN guidelines recommend EBRT and androgen deprivation therapy (i.e., HT), EBRT and brachytherapy with/without HT, EBRT and HT plus docetaxel chemotherapy, or RP and pelvic lymph nodal dissection. ${ }^{4}$ Grimm et al. ${ }^{16}$ evaluated the relative effectiveness of RP and RT for high-risk PC, and found that RT plus brachytherapy with/without HT appeared superior to more localized treatments, such as RP or RT alone. Furthermore, an RT dose of 70 Gy might be inadequate to eradicate high-risk PC, as a randomized dose escalation trial (78 Gy vs. $70 \mathrm{~Gy}$ ) revealed that the higher dose was associated with a better 5 -year BCRFS rate $(78 \%$ vs. $68 \%, p=0.03)$, especially among patients with pretreatment PSA levels of $>10-20$ $\mathrm{ng} / \mathrm{mL}$ (87.5\% vs. $67.6 \%, \mathrm{p}=0.437) .{ }^{17}$ Moreover, a randomized trial of HT plus external beam RT revealed that patients with locally advanced PC experienced favorable survival outcomes. ${ }^{18}$ However, the efficacies of EBRT and RP have not been compared in randomized trials, especially among patients with high-risk (or locally advanced) PC, which has forced clinicians to rely on observational data.

Previous studies have suggested that nearly identical control of high-risk PC is provided by RP and RT with appropriate NHT. ${ }^{19}$ Nevertheless, there are variable definitions of BCR, which makes it difficult to accurately compare outcomes across modalities using PSA endpoints. ${ }^{20,21}$ Nevertheless, the present study revealed that RT provided significantly superior BCRFS among patients with high-risk or locally advanced PC $(p=0.004)$ (Table 2, Fig. 2). It is possible that this difference is related to the time needed to reach the nadir PSA level in the two groups, as the nadir PSA level may not be observed for 1-2 years (occasionally longer) among patients receiving RT. In contrast, almost all RP-treated patients will achieve the nadir PSA level within 1-2 months after treatment. Thus, clin- ically meaningful and reliable results may require longer follow-up periods than the follow-up for the present study. The RT group experienced significantly inferior CPFS $(p=0.013)$ (Table 2, Fig. 2). In this context, bowel and bladder complications are common after RT, especially among patients who are treated using 3D-CRT (vs. intensity-modulated RT). ${ }^{22,23}$ Furthermore, Cicchetti et al. ${ }^{24}$ reported pooled results from the Airopros 0102 and TROG03.04 RADAR trials, which involved prescribed 3D-CRT doses (66-80 Gy; median, $73.2 \mathrm{~Gy}$; interquartile range, 70-75 Gy; 1.8-2 Gy/day) that were comparable to the present study's prescribed doses (70-76 Gy, 2 Gy/day). In those trials, large rectal volumes that received mid-range doses (30-50 Gy) were associated with rectal pain. Thus, Cicchetti et al. ${ }^{24}$ concluded that sparing the anorectal area from low-to-mid-range doses may reduce the incidence of rectal pain. This may explain why the present study detected better CPFS in the RP group, compared to the RT group.

The RT group always underwent HT before and after RT, whereas NHT is typically used as a standard therapy without surgery. Nevertheless, it is difficult to surgically remove high-risk PC tumors with negative margins, given the large tumor burden, and NHT may help reduce the prostate's size and control the surgical margins. For example, several studies have indicated that NHT before RP reduced the rate of positive surgical margins, which potentially resulted in complete pathological responses. ${ }^{25}$ However, differences in the 2 groups' characteristics (e.g., physical condition, staging, and hormonal duration and agent) make it difficult to compare these outcomes, despite significant differences being observed for BCR and CPFS (Fig. 2). Moreover, NHT has not been confirmed to be beneficial for patient outcomes, especially in terms of PSA-free survival in randomized trials. ${ }^{26}$ Among patients with high-risk PC, the reported rates of PSA-free survival after RP alone were $35 \%-62 \%$, which are similar to the rate from the present study. In addition, a systematic review of adjuvant HT plus NHT with $\mathrm{RP}$ or RT for high risk or locally advanced PC revealed that NHT before RP did not significantly improve OS (odds ratio, $1.11 ; \mathrm{p}=0.69){ }^{27}$ Nevertheless, it did provide significant improvements in the positive surgical margin rate, lymph node involvement, pathological staging, and organ confinement (all $\mathrm{p}<$ $0.05)$. However, the outcomes were not significantly different between RP and RT after NHT, with the exception of significantly better CPFS in the RP group.

Other systematic reviews have revealed significant hetero- 
geneity in the outcomes for NHT and adjuvant HT after either RT or RP. For example, some studies have indicated that NHT before RT significantly improved OS among patients with a Gleason score of 2-6, although 2 studies failed to detect significant improvement in disease-specific survival. However, adjuvant HT plus RP or RT was associated with significant clinical benefits among patients with localized high risk or locally advanced PC. ${ }^{28}$ Thus, adjuvant HT may provide significant local control after RP or RT, which could improve the patient's quality of life. It is also possible that it could provide a survival advantage, as adjuvant HT after RT provided significantly improved OS at 5 years and 10 years, as well as significant improvements in disease-specific survival and disease-free survival at 5 years. Adjuvant HT after RP also provided significant improvements in disease-free survival and BCR-free survival at 5 years and 10 years, although the results were not statistically significant for OS at 5 years. Furthermore, a subgroup of patients with undifferentiated carcinoma has a high risk of progression after RP, and these patients should receive adjuvant HT or a different therapy.

It remains unclear whether adjuvant RT or NHT are effective after RP, and these therapies should only be evaluated in clinical trials. In addition, HT is associated with significant side effects, such as hot flushes and gynecomastia, as well as a high cost. Thus, the decision to use HT must be made in collaboration between patients, clinicians, and policy makers, and should account for the treatment's potential benefits, toxicity, and cost. Although adjuvant HT can improve prognosis, NHT did not significantly improve the prognosis for high-risk PC. Thus, additional research is needed regarding HT choice, duration, and scheduling, which should consider the long-term effects of patient's quality of life and potential toxicity.

In our study, the RT group showed better 5-year OS rate than the RP group. The authors presume that the reason for this is due to the fact that the number of RP group was higher and the FU duration was longer than RT group. As a result, patients who became censored were more likely to have RP group than RT group. However, the number of deaths of RP group is lower than RT group. Therefore, if the FU duration becomes longer and the number of patients increases, the OS rate may change. In addition, significant downstaging (pT3N0+pT4N0=2.03\%) and a positive surgical margin (PSM) rate of $4.6 \%$ were demonstrated in the neoadjuvant HT before the RP group, as shown in Table 1 . In one previous study, ${ }^{29} 56 \%$ of pT3-4 and $49 \%$ of PSM rate were reported in RP without neoadjuvant HT.

The present study has several limitations. First, the retrospective design and small sample size are associated with risks of selection bias. Those patients received RT and transferred to other hospitals for the follow-up were excluded at the early enrollment. Second, the RP group had received NHT, while the RT group had received NHT and adjuvant HT. Third, the study was not able to evaluate data regarding health-related quality of life (e.g., continence and erectile function), which could affect treatment selection. Nevertheless, the present study's results may help improve the management of patients with high-risk PC, based on the lack of prospective randomized clinical trials.

\section{CONCLUSIONS}

The present study revealed that, among patients with highrisk PC previously treated using NHT, the RP group experienced better CPFS than the RT group after adjusting for clinical TN stage.

\section{CONFLICT OF INTEREST}

The authors claim no conflicts of interest.

\section{REFERENCES}

1. Ferlay J, Shin HR, Bray F, Forman D, Mathers C, Parkin DM. Estimates of worldwide burden of cancer in 2008: GLOBOCAN 2008. Int J Cancer 2010;127:2893-917.

2. Heidenreich A, Bastian PJ, Bellmunt J, Bolla M, Joniau S, van der Kwast $\mathrm{T}$, et al. EAU guidelines on prostate cancer. Part II: treatment of advanced, relapsing, and castration-resistant prostate cancer. Eur Urol 2014;65:467-79.

3. National Comprehensive Cancer Network. NCCN Guidelines for Patients: Prostate Cancer. ver 3. Fort Wathington (PA): National Comprehensive Cancer Network; 2016.

4. Carroll PR, Parsons JK, Andriole G, Bahnson RR, Castle EP, Catalona WJ, et al. NCCN Guidelines insights: prostate cancer early detection, version 2.2016. J Natl Compr Canc Netw 2016;14:509-19.

5. Pompe RS, Karakiewicz PI, Tian Z, Mandel P, Steuber T, Schlomm T, et al. Oncologic and functional outcomes after radical prostatectomy for high or very high risk prostate cancer: European Validation of the Current $\mathrm{NCCN} 囚$ Guideline. J Urol 2017;198:354-61.

6. Shelley MD, Kumar S, Wilt T, Staffurth J, Coles B, Mason 
MD. A systematic review and meta-analysis of randomised trials of neo-adjuvant hormone therapy for localised and locally advanced prostate carcinoma. Cancer Treat Rev 2009;35: 9-17.

7. Schulman CC, Debruyne FM, Forster G, Selvaggi FP, Zlotta AR, Witjes WP. 4-Year follow-up results of a European prospective randomized study on neoadjuvant hormonal therapy prior to radical prostatectomy in T2-3N0M0 prostate cancer. European Study Group on Neoadjuvant Treatment of Prostate Cancer. Eur Urol 2000;38:706-13.

8. Zilli T, Dal Pra A, Kountouri M, Miralbell R. Prognostic value of biochemical response to neoadjuvant androgen deprivation before external beam radiotherapy for prostate cancer: A systematic review of the literature. Cancer Treat Rev 2016;46: 35-41.

9. Hoffman RM, Koyama T, Fan KH, Albertsen PC, Barry MJ, Goodman M, et al. Mortality after radical prostatectomy or external beam radiotherapy for localized prostate cancer. J Natl Cancer Inst 2013;105:711-8.

10. Epstein JI, Allsbrook WC Jr, Amin MB, Egevad LL; ISUP Grading Committee. The 2005 International Society of Urological Pathology (ISUP) Consensus Conference on Gleason Grading of Prostatic Carcinoma. Am J Surg Pathol 2005;29:1228-42.

11. Kim YJ, Cho KH, Pyo HR, Lee KH, Moon SH, Kim TH, et al. Radical prostatectomy versus external beam radiotherapy for localized prostate cancer: Comparison of treatment outcomes. Strahlenther Onkol 2015;191:321-9.

12. Cheung R. In regards to Roach et al. defining biochemical failure following radiotherapy with or without hormonal therapy in men with clinically localized prostate cancer: recommendations of the RTOG-ASTRO Phoenix Consensus Conference (Int J Radiat Oncol Biol Phys 2006;65:965-974). Int J Radiat Oncol Biol Phys 2006;66:1274.

13. Gillessen S, Attard G, Beer TM, Beltran H, Bossi A, Bristow $\mathrm{R}$, et al. Management of patients with advanced prostate cancer: the report of the Advanced Prostate Cancer Consensus Conference APCCC 2017. Eur Urol 2018;73:178-211.

14. Torre LA, Siegel RL, Ward EM, Jemal A. Global cancer incidence and mortality rates and trends--an update. Cancer Epidemiol Biomarkers Prev 2016;25:16-27.

15. van den Ouden D, Schröder FH. The treatment of locally advanced (T3) prostatic carcinoma using radical prostatectomy or radiotherapy. A review. Tijdschr Gerontol Geriatr 1998; 29:74-9.

16. Grimm P, Billiet I, Bostwick D, Dicker AP, Frank S, Immerzeel $\mathrm{J}$, et al. Comparative analysis of prostate-specific antigen free survival outcomes for patients with low, intermediate and high risk prostate cancer treatment by radical therapy. Results from the Prostate Cancer Results Study Group. BJU Int 2012;109 Suppl 1:22-9.

17. Pollack A, Zagars GK, Smith LG, Lee JJ, von Eschenbach
AC, Antolak JA, et al. Preliminary results of a randomized radiotherapy dose-escalation study comparing 70 Gy with 78 Gy for prostate cancer. J Clin Oncol 2000;18:3904-11.

18. Bolla M, Gonzalez D, Warde P, Dubois JB, Mirimanoff RO, Storme $\mathrm{G}$, et al. Improved survival in patients with locally advanced prostate cancer treated with radiotherapy and goserelin. N Engl J Med 1997;337:295-300.

19. Gomella LG, Zeltser I, Valicenti RK. Use of neoadjuvant and adjuvant therapy to prevent or delay recurrence of prostate cancer in patients undergoing surgical treatment for prostate cancer. Urology 2003;62 Suppl 1:46-54.

20. Cooperberg MR, Vickers AJ, Broering JM, Carroll PR. Comparative risk-adjusted mortality outcomes after primary surgery, radiotherapy, or androgen-deprivation therapy for localized prostate cancer. Cancer 2010;116:5226-34.

21. Cookson MS, Aus G, Burnett AL, Canby-Hagino ED, D'Amico AV, Dmochowski RR, et al. Variation in the definition of biochemical recurrence in patients treated for localized prostate cancer: the American Urological Association Prostate Guidelines for Localized Prostate Cancer Update Panel report and recommendations for a standard in the reporting of surgical outcomes. J Urol 2007;177:540-5.

22. Prabhakar R, Oates R, Daryl J, Chang J, Geso M, Cramb J. Rectal complication probability from composite volumes derived from daily cone beam computed tomography in prostate cancer radiotherapy. J Cancer Res Ther 2016;12:374-8.

23. Wortel RC, Witte MG, van der Heide UA, Pos FJ, Lebesque $\mathrm{JV}$, van Herk M, et al. Dose-surface maps identifying local dose-effects for acute gastrointestinal toxicity after radiotherapy for prostate cancer. Radiother Oncol 2015;117:51520.

24. Cicchetti A, Rancati T, Ebert M, Fiorino C, Palorini F, Kennedy A, et al. Modelling late stool frequency and rectal pain after radical radiotherapy in prostate cancer patients: Results from a large pooled population. Phys Med 2016;32: 1690-7.

25. Bono AV, Pagano F, Montironi R, Zattoni F, Manganelli A, Selvaggi FP, et al. Effect of complete androgen blockade on pathologic stage and resection margin status of prostate cancer: progress pathology report of the Italian PROSIT study. Urology 2001;57:117-21.

26. Soloway MS, Pareek K, Sharifi R, Wajsman Z, McLeod D, Wood DP Jr, et al. Neoadjuvant androgen ablation before radical prostatectomy in cT2bNxMo prostate cancer: 5-year results. J Urol 2002;167:112-6.

27. Fahmy O, Khairul-Asri MG, Hadi SHSM, Gakis G, Stenzl A. The role of radical prostatectomy and radiotherapy in treatment of locally advanced prostate cancer: a systematic review and meta-analysis. Urol Int 2017;99:249-56.

28. Kumar S, Shelley M, Harrison C, Coles B, Wilt TJ, Mason MD. Neo-adjuvant and adjuvant hormone therapy for lo- 
134 대한비뇨기종양학술지 : 제 16 권 제 3 호 2018

calised and locally advanced prostate cancer. Cochrane Database Syst Rev 2006;(4):CD006019.

29. Ward JF, Slezak JM, Blute ML, Bergstralh EJ, Zincke H.
Radical prostatectomy for clinically advanced (cT3) prostate cancer since the advent of prostate-specific antigen testing: 15-year outcome. BJU Int 2005;95:751-6. 